\title{
THE FOURTEENTH REGULAR MEETING OF THE SOUTHWESTERN SECTION
}

The fourteenth regular meeting of the Southwestern Section of the American Mathematical Society was held at Washington University on Saturday, November 26, 1921. The attendance included the following twenty members:

Ammerman, Ashton, Dunkel, W. W. Hart, Hedrick, Ingold, Lefschetz, Luby, Lytle, McKelvey, U. G. Mitchell, Rider, Roever, Slaught, Edwin R. Smith, Eugene Stephens, Stouffer, E. A. Weeks, Westfall, J. M. Young.

The afternoon session was a joint meeting with the Missouri Section of the Mathematical Association of America. Professor Hedrick occupied the chair during the morning session and was relieved during the afternoon session by Professor Ingold, chairman of the Missouri Section.

It was voted to hold the next meeting of the Southwestern Section at the University of Kansas. The following program committee was elected: Professors Ashton (chairman), Brenke, and Stouffer (secretary).

The titles and abstracts of the papers read at this meeting follow below. The papers of Dr. Kendall and Professor Chittenden were read by title.

1. Dr. Claribel Kendall: Certain congruences determined by a given surface.

In this paper a surface is considered as defined by a pair of partial differential equations of the second order as is done by Professor Wilczynski in his first memoir on the projective differential geometry of curved surfaces (TRANsactions of THIs SocIeTr, vol. 8). A line $l$ is determined by two points which are defined with respect to a local tetrahedron of reference and in terms of the parameters of the surface. Thus a line is given for every point on the surface and hence a congruence is determined. Relative to this general congruence, formulas are found (1) for the torsal curves on the surface, i.e., the curves which give the developables of the congruence, (2) 
for a new class of curves which are called the guide curves of the congruence, (3) for the focal points of the line $l$. These formulas are applied to special congruences and various relations among the lines generating these congruences are found.

2. Professor Otto Dunkel: The direct determination of the minimum area between a curve and its caustic.

In the Washington University Studies, Scientific SerIes, vol. 8, no. 2, pp. 183-194, necessary and sufficient conditions were derived for the existence of a minimum area by methods similar to the usual methods of the calculus of variations. The present treatment by Professor Dunkel is similar to the one given by him for the solution of Euler's problem (American Mathematical Monthly, vol. 28, pp. 15-19) in that advantage is taken of the fact that the integrand may be reduced to a square and that the problem is solved by elementary means without the use of the general theory of the calculus of variations. The solution of this problem differs from that of Euler's problem, notably in that most of the results are obtained by reductions to complete squares.

3. Professors E. R. Hedrick, Louis Ingold, and W. D. A. Westfall: Classification of non-analytic functions.

In this paper Beltrami's extensions of the Cauchy-Riemann equations are made the basis for a classification of all nonanalytic functions. Two functions belong to the same class if the fundamental quantities $E, F, G$, for the two functions are proportional. Some properties of functions belonging to the same class are then developed.

4. Professor S. Lefschetz: A topological group of algebraic varieties.

The group in question is the transformation group $G$ of the $(d-1)$-dimensional cycles of a linear pencil of hypersurfaces of a $d$-dimensional variety. The singularities being ordinary, the operations of $G$ acting upon a given cycle are completely defined by means of Kronecker characteristics (the algebraic number of intersections of cycles). For $d$ even the group is of the same type as the Picard group, defined by him for $d=2$. For $d$ odd the fundamental substitutions are of order two, $G$ being nevertheless infinite. 
5. Professor S. Lefschetz: On multiply periodic functions.

Given a period matrix for $2 p$-ply periodic meromorphic functions, we may define for the variables a fundamental domain in the form of a $2 p$-dimensional generalized ring. The zeros common to $p-1$ suitable periodic functions define a two-cycle of the ring. This cycle can be readily expressed in terms of those of a fundamental set. Several results are obtained, the most important being the Picard-Poincaré existence theorem for the matrix, and the classification of all intermediary functions in terms of a certain fundamental set.

6. Professor W. H. Roever: An application of the theorem of Jacobi.

In this paper Professor Roever applies the theorem of Jacobi* to the integration of the differential equations of motion of a particle referred to a rotating frame of reference, when a constant force acts parallel to the axis of rotation.

7. Professor E. B. Stouffer: On curves on singular ruled surfaces.

A ruled surface in a space of five dimensions is said to be singular if every set of three consecutive generators lies in. a space of four dimensions. On every such singular ruled surface there is a curve along which tangents to the surface intersect three consecutive generators. Moreover, this curve is unique unless the ruled surface lies entirely in a space of three dimensions. A system of three linear homogeneous differential equations is used for the study of these forms.

8. Professor E. W. Chittenden: On the division of a plane by a set of points.

The following theorem is proved. Suppose $K$ is a plane point set, $S$ is the set of all points of the plane, while $S-K=S_{1}+S_{2}$, where $S_{1}$ and $S_{2}$ are two mutually exclusive domains such that every point of $K$ is a common limit point of $S_{1}$ and $S_{2}$. Then the set $K$ is closed and connected. That $K$ is connected was shown by J. R. Kline under the added restriction that $K$ be connected "im kleinen" (Transactions of this Society, vol. 21 (1920), p. 452, Lemma B).

E. B. Stouffer, Secretary of the Section.

*For a statement of this theorem see, for instance, Appell, Traité de Mécanique Rationnelle, 4th edition, vol. 1, p. 560. 